\title{
La brecha económica entre los modelos teóricos y su aplicación en la frontera méxico-norteamericana
}

Jerry R. Ladman

Arizona State University

\section{Resumen}

Este artículo tomará brevemente en consideración el modelo teórico "multiplicador de las ciudades gemelas" que describe la interdependencia proveniente del intercambio económico entre regiones fronterizas contiguas y demuestra cómo factores económicos, endógenos y exógenos a la frontera afectan ambas regiones. Este modelo sirve para conceptualizar y describir la naturaleza interdependiente de las relaciones económicas en las regiones contiguas, y si son aplicados, podrían ser útiles para predecir variaciones en las dos economías. Sin embargo, la aplicación de dichos modelos para predecir cambios en la actividad económica, requieren una cantidad considerable de datos muy costosos tanto en tiempo cono en dinero, además de que deben ser actualizados para que sean válidos.

Palabras clave: brecha económica, modelos teóricos, interdependencia, regiones fronterizas.

\begin{abstract}
This article will briefly consider the theoretical model "multiplying factor of twin cities" which describes the interdependence resulting from the economical exchange between adjacent border regions and shows how economic, endogenous, and exogenous border factors are affecting both regions. This model is useful to conceptualize and describe the interdependent nature of the economical relations in the contiguous regions, which; if properly applied, they could be useful to predict the variations of the two economics. However, using these models to predict changes on the economic activity requires money and time, but also a considerable and expensive amount of information, which should be updated in order to be valid.
\end{abstract}

Keywords: economic breach, theoretical models, interdependence, border regions. 


\title{
LA BRECHA ECONOMICA ENTRE LOS MODELOS TEORICOS Y SU APLICACION EN LA FRONTERA MEXICO-NORTEAMERICANA*
}

\author{
Por \\ Jerry R. Ladman \\ Director del Center for Latin American Studies \\ Arizona State University, Tempe, Arizona
}

La economía de la región fronteriza mexico-norteamericana es única para los dos países. Está caracterizada por un largo número de contiguas regiones económicas envolviendo centros de población localizados a cada lado de la frontera y separados por un límite internacional. Muchas veces, estas regiones han crecido alrededor de una base económica derivada de los recursos naturales, como minería o agricultura con irrigación artificial. En otros, se han desarrollado principalmente por ser centros comerciales o turísticos. Debido a su relativo aislamiento de los principales centros económicos de los dos países, así como a la larga historia de relaciones económicas entre las dos naciones, incluyendo flujos relativamente irrestrictos de productos y recursos, las economías regionales contiguas han crecido acostumbradas a considerables intercambios económicos. Consecuentemente, debido a estas ligaduras, sucesos que afectan las condiciones económicas en un lado de la frontera tienen impactos importantes, no sólo en la esa región fronteriza sino también en la del otro país.

Durante 1979 elaboré un modelo teórico llamado "el multiplicador de las ciudades gemelas", que describe la interdependencia proveniente del intercambio económico entre regiones fronterizas contiguas, y demuestra cómo factores económicos, endógenos o exógenos a la frontera, afectan ambas regiones. Estos modelos sirven para conceptualizar y describir la naturaleza interdependiente de las relaciones económicas en las regiones contiguas y, si son aplicados, podrían ser útiles para predecir variaciones en las dos econom ías, cuando cambien las condiciones o las políticas económicas. Ha habido pocos intentos de aplicar estos modelos, principalmente debido al alto costo y dificultad en la obtención de datos adecuados y actualizados. En este artículo consideraré brevemente el modelo, examinaré los problemas de su aplicación y abogaré por la necesidad de la adecuada recolección de datos.

\footnotetext{
*Traducción del artículo en inglés titulado "The gap between theoretical modeling and the application of these models to the U.S. - Mexican border economy", editado en Statistical abstract of the United States-Mexico bordelarlands, eds. James W. Wilke and Peter Reich, publicado por el Centro Latinoamericano de la Universidad de California en Los Angeles. El permiso para la publicación de este artículo es otorgado por los Regentes de la Universidad de California, que poseen los derechos de propiedad. El autor agradece a Walter E. Quiroga Lanz por la traduccion.
} 


\section{MODELO}

Desarrollando originalmente por Ladman ${ }^{1}$ y refinado por Ladman y Duffy ${ }^{2}$, el modelo combina el análisis de los multiplicadores de ingreso regional y comercio exterior para mostrar el nivel de equilibrio de la actividad económica en dos economías fronterizas regionales contiguas, A y B, como función de los niveles autónomos de gasto de las dos economías, y toma en cuenta los nexos entre las dos economías que surgen del gasto inducido. El modelo muestra cómo las dos economías se ajustan a cambios en los niveles de gasto autónomo o bien a factores que causan gasto inducido en cualquier región. Además, el modelo puede ser usado como marco analítico para examınar históricamente el desarrollo económico de una región fronteriza entre dos países.

Los gastos autónomos en A o B pueden provenir de varias fuentes. Ejemplos típicos son: gastos de gobierno en proyectos, inversiones del sector privado en negocios, y producción de bienes y servicios para exportación de la región. Debido a los gastos inducidos dentro de cada renglón, los gastos autónomos en $\mathrm{A}(\mathrm{B})$ dan lugar a un nivel de ingreso de equilibrio, $\mathrm{Y}_{\mathrm{A}}\left(\mathrm{Y}_{\mathrm{B}}\right)$, que es mayor que el gasto autónomo original. Este incremento es debido al efecto del multiplicador directo y depende de la proporción del ingreso ganado, que tiende a ser nuevamente gastado en la región.

Existe otro multiplicador, el multiplicador cruzado, que se desarrolla debido a que en dos regiones contiguas los ingresos personales de una tienden a ser, en parte, gastados en la segunda. De este modo, el multiplicador cruzado influencia a $Y_{A}$ así como a $Y_{B}$. Cuando el ingreso en $A(B)$ sube o baja debido a un cambio en el nivel de gasto autónomo en $\mathrm{A}(\mathrm{B})$, habrá un impacto negativo o positivo en $\mathrm{Y}_{B}\left(\mathrm{Y}_{\mathrm{A}}\right)$, en cuanto las compras en $\mathrm{B}(\mathrm{A})$ por residentes de $A(B)$ incrementen o disminuyan. Debido a que una porción de ese ingreso ganado como resultado de esas compras en $\mathrm{B}(\mathrm{A})$ será nuevamente gastado en la otra economía, habrán incrementos o decrementos en $\mathrm{Y}_{\mathrm{A}} \mathrm{o}$ bien en $\mathrm{Y}_{\mathrm{B}}$ debido al efecto del multiplicador cruzado.

Por lo tanto, los niveles totales de $\mathrm{Y}_{\mathrm{A}} \mathrm{y}$ de $\mathrm{Y}_{\mathrm{B}}$ dependerán del volumen inicial del gasto autónomo en A y/o, B, así como también del tamaño de los multiplicadores directo y cruzado en A y B. Los valores de equilibrio de $\mathrm{Y}_{\mathrm{A}}$ y de $\mathrm{Y}_{\mathrm{B}}$ son demostrados en las ecuaciones 1 y 2.

\footnotetext{
1Jerry R. Ladman, "The economic interdependence of contiguous border cities: the twin city multiplier", The annals of regional science, v. 13 No. 1 Niarch, 1979.

${ }^{2}$ Jerry R. Ladman y Michael K. Duffy, "Intercambio económico en el área fronteriza méxico-norteamericana: comercio y turismo", Estudios fronterizos. Ciudad de México: ANUIES, 1981 pp. 185-206.
} 
1. $\mathrm{Y}_{\mathrm{A}}=\mathrm{D}_{\mathrm{A}} \mathrm{S}_{\mathrm{A}}+\mathrm{R}_{\mathrm{A}} \mathrm{S}_{\mathrm{B}}$

2. $\mathrm{Y}_{\mathrm{B}}=\mathrm{R}_{\mathrm{B}} \mathrm{S}_{\mathrm{A}}+\mathrm{D}_{\mathrm{B}} \mathrm{S}_{\mathrm{B}}$

En estas ecuaciones, $D_{A}\left(D_{B}\right), R_{A}\left(R_{B}\right), y S_{A}\left(S_{B}\right)$ corresponden al multiplicador directo, multiplicador cruzado, $y$ a la suma de gastos autónomos en $A(B)$ respectivamente.

Las dos ecuaciones pueden ser usadas para mostrar los efectos de cualquier cambio, o combinación de cambios, del nivel de gastos autónomos en los niveles de $\mathrm{Y}_{\mathrm{A}}$ y de $\mathrm{Y}_{\mathrm{B}}$. Por ejemplo, si existiese un incremento (decremento) en gastos autónomos $\Delta \mathrm{S}_{\mathrm{A}}$ en la región $\mathrm{A}$, el impacto en las dos regiones $\left(\Delta \mathrm{Y}_{\mathrm{A}}\right.$ y $\left.\Delta \mathrm{Y}_{\mathrm{B}}\right)$ puede ser demostrado en la ecuación 3. De un modo similar, el efecto de $\Delta \mathrm{S}_{\mathrm{B}}$ podría ser representado:

$$
\text { 3. } \begin{aligned}
\Delta \mathrm{Y}_{\mathrm{A}} & =\mathrm{D}_{\mathrm{A}}\left(\Delta \mathrm{S}_{\mathrm{A}}\right) \\
\Delta \mathrm{Y}_{\mathrm{B}} & =\mathrm{R}_{\mathrm{B}}\left(\Delta \mathrm{S}_{\mathrm{A}}\right)
\end{aligned}
$$

Las anteriores ecuaciones están presentadas en forma resumida. Cuando son desarrolladas se vuelven mucho más complejas, como se demuestra en las ecuaciones 4 a 9.3

$$
\begin{aligned}
& \text { 4. } \mathrm{S}_{\mathrm{A}}=\overline{\mathrm{c}_{\mathrm{A}}}+\overline{\mathrm{T}}_{\mathrm{A}}+\overline{\mathrm{G}}_{\mathrm{A}}+\left(\overline{\mathrm{m}}_{\mathrm{B}}^{\mathrm{A}}+\overline{\mathrm{X}}_{\mathrm{A}}^{\mathrm{R}}\right)-\left(\overline{\mathrm{m}}_{\mathrm{A}}^{\mathrm{B}}+\overline{\mathrm{m}}_{\mathrm{B}}^{\mathrm{R}}\right)+ \\
& {\left[\left(m_{A}^{B}+m_{A}^{R}\right)-c_{A}\right] \bar{T}_{A}-m_{B}^{A} \bar{T}_{B} \text {, }} \\
& \text { 5. } \overline{\mathrm{S}}_{\mathrm{B}}=\mathrm{c}_{\mathrm{B}}+\overline{\mathrm{I}}_{\mathrm{B}}+\overline{\mathrm{G}}_{\mathrm{B}}+\left(\overline{\mathrm{m}}_{\mathrm{A}}^{\mathrm{B}}+\overline{\mathrm{X}}_{\mathrm{B}}^{\mathrm{R}}\right)-\left(\overline{\mathrm{m}}_{\mathrm{B}}^{\mathrm{A}}+\overline{\mathrm{m}}_{\mathrm{B}}^{\mathrm{R}}\right)+ \\
& {\left[\left(m_{B}^{A}+m_{B}^{R}\right)-c_{B}\right] \bar{T}_{B}-m_{A}^{B} \bar{T}_{A} \text {, }} \\
& \mathrm{s}_{\mathrm{B}}+\left(\mathrm{m}_{\hat{B}}^{\hat{B}}+\mathrm{m}_{\mathrm{B}}^{\mathrm{R}}\right)
\end{aligned}
$$

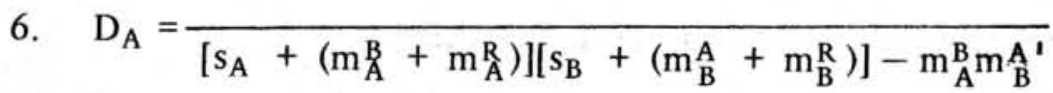

$$
\mathrm{m}_{\mathrm{B}}^{\mathrm{A}}
$$

3Para una elaboración completa del modelo, el lector debe remitirse a Ladman y Duffy, "Intercambio econó mico en el área fronteriza méxico-norteamericana: comercio y turismo". 
7. $\mathrm{R}_{\mathrm{A}}=\frac{}{\left[\mathrm{s}_{\mathrm{A}}+\left(\mathrm{m}_{\mathrm{A}}^{\mathrm{B}}+\mathrm{m}_{\mathrm{A}}^{\mathrm{R}}\right)\right]\left[\mathrm{s}_{\mathrm{B}}+\left(\mathrm{m}_{\mathrm{B}}^{\mathrm{A}}+\mathrm{m}_{\mathrm{B}}^{\mathrm{R}}\right)\right]-\mathrm{m}_{\mathrm{A}}^{\mathrm{B}} \mathrm{m}_{\mathrm{B}}^{\mathrm{A}^{\prime}}}$ $\mathrm{m}_{\AA}^{\mathrm{B}}$

8. $\mathrm{R}_{\mathrm{B}}=\frac{}{\left[\mathrm{s}_{\mathrm{A}}+\left(\mathrm{m}_{\mathrm{A}}^{\mathrm{B}}+\mathrm{m}_{\mathrm{A}}^{\mathrm{R}}\right)\right]\left[\mathrm{s}_{\mathrm{B}}+\left(\mathrm{m}_{\mathrm{B}}^{\mathrm{A}}+\mathrm{m}_{\mathrm{B}}^{\mathrm{R}}\right)\right]-\mathrm{m}_{\mathrm{A}}^{\mathrm{B}} \mathrm{m}_{\mathrm{B}}^{\mathrm{A}^{\prime}}}$

$$
s_{A}+\left(m_{A}^{B}+m_{A}^{R}\right)
$$

9. $\mathrm{D}_{\mathrm{B}}=\frac{}{\left[\mathrm{s}_{\mathrm{A}}+\left(\mathrm{m}_{\mathrm{A}}^{\mathrm{B}}+\mathrm{m}_{\mathrm{B}}^{\mathrm{R}}\right)\right]\left[\mathrm{s}_{\mathrm{B}}+\left(\mathrm{m}_{\mathrm{B}}^{\mathrm{A}}+\mathrm{m}_{\mathrm{B}}^{\mathrm{R}}\right)\right]-\mathrm{m}_{\mathrm{A}}^{\mathrm{B}} \mathrm{m}_{\mathrm{B}}^{\mathrm{A}^{4}}}$

En las anteriores ecuaciones, las letras acompañadas por una barra denotan gasto autónomo. Para una región $\mathrm{A}, \mathrm{C}_{\mathrm{A}}$ representa consumo, $\mathrm{I}_{\mathrm{A}}$ representa inversión, $G_{A}$ gastos del gooierno, $M_{B}^{A}$ ventas de exportación de $A$ a $B, X_{A}^{R}$ ventas de exportación de $A$ al resto del mundo (definidas como fuera de $A$ y $B$ ), $M_{A}^{B}$ compras de importación de $B$ a $A, M_{A}^{R}$ compras del resto del mundo a $\mathrm{A}, \mathrm{y} \mathrm{T}_{\mathrm{A}}$ impuestos en $\mathrm{A}$. Existen variables correspondientes para la región $\mathrm{B}$.

En el modelo existen parámetros de comportamiento que relacionan los gastos autónomos y los niveles de ingreso de equilibrio. Estos parámetros determinan el tamaño de los multiplicadores porque dan lugar a gastos inducidos. En el modelo existen variables sin barra, que corresponden a las propensiones marginales. Para la región $\mathrm{A}, \mathrm{C}_{\mathrm{A}}$ representa la propensión marginal a consumir; $\mathrm{S}_{\mathrm{A}}$ es la propensión marginal al ahorro; $\mathrm{M}_{\mathrm{A}}^{\mathrm{B}}$ es la propensión marginal a importar de $\mathrm{B}$ hacia $\mathrm{A}, \mathrm{y} \mathrm{M}_{\mathrm{A}}^{\mathrm{R}}$ es la propensión marginal de importar del resto del mundo hacia A. Existen variables correspondientes para la región $\mathrm{B}$.

Si uno o varios parámetros cambian existiría un impacto correspondiente en $\mathrm{Y}_{\mathrm{A}} \mathrm{y}$ en $\mathrm{Y}_{\mathrm{B}}$, cuyos efectos pueden ser demostrados por el modelo.

\section{PROBLEMAS EN LA APLICACION DEL MODELO}

La aplicación de este modelo ofrece un potencial considerable en demostrar el impacto de nuevas políticas económicas o cambios en las condiciones económicas que tienen como resultado nuevos gastos autónomos o variaciones en los parámetros, ya sea en $\mathrm{A}$ y $\mathrm{B}$ o en $\mathrm{Y}_{\mathrm{A}}$ y en $\mathrm{Y}_{\mathrm{B}}$. Existen, sin embargo, dos problemas. 
El primer problema es que el modelo está expresado en términos reales y no monetarios. Cuando el modelo fue desarrollado, este aspecto no fue considerado como un defecto muy importante debido a que la tasa de cambio entre las monedas norteamericana y mexicana era virtualmente constante. Sin embargo, la situación ha cambiado en los últimos años, especialmente desde 1982, cuando la tasa de cambio fluctuó considerablemente. Los cambios en los precios relativos de los bienes en las dos regiones afectarán las propensiones marginales de los residentes de A y B en los gastos de consumo inducido en su región, así como también en las importaciones de otras regiones y del resto del mundo. Del mismo modo, cambios en los precios pueden también impactar en la propensión marginal al ahorro, y todavía más, estos cambios pueden impactar en gastos autónomos como aquellos de turismo, inversiones en manufactura e inversiones de gobierno o transferencias.

Eì modelo puede ser ajustado para poder tomar en cuenta estos cambios. El hacerlo, sin embargo, lo haría muy complicado y requeriría conocimientos detallados de las relaciones entre los cambios en precios relativos a aquellos en gastos de consumo inducido, ahorros, y gastos autónomos. Más aún, la obtención de este conocimiento sería costosa en tiempo y dinero.

El segundo problema es la obtención de datos adecuados. Sería necesario observar y especificar los cambios en gastos autónomos. Para nuevos proyectoś que requieren inversión fija y nómina de salarios esto no sería difícil. Sin embargo, para gastos autónomos que emanan de la producción para exportación, turismo o actividades de importación-exportación, las cantidades dependen de factores exógenos a las regiones, los cuales deben ser considerados en la estimación del monto de dichos gastos en las dos regiones. Esto requeriría conocimiento y datos acerca de estas relaciones y de su efecto en los gastos de las dos regiones.

Un aspecto más serio del problema es la estimación de los parámetros del modelo que actúan como gastos inducidos; por ejemplo, todas las propensiones marginales, muchas de las cuales son asumidas para A y B en el modelo. Virtualmente es inexistente el modo exacto de obtener esta información, a no ser a través de largas muestras por medio de encuestas en ambas poblaciones. Realizada correctamente, la muestra debe ser estratificada para tomar en cuenta las diferentes clases sociales y de ingreso. Estas encuestas consumen mucho tiempo y son excesivamente costosas. Más aún, debido a los cambios en precios relativos, estos deben ser puestos al día regularmente para tomar en cuenta el efecto en los parámetros de cambios en la tasa de cambio. Adicionalmente, cambios estructurales en las dos economías, incluyendo revisiones en las leyes impositivas y regulaciones gubernamentales, así como la relativa disponibilidad o calidad de los bienes, causarán cambios en los parámetros y deberán ser tomadas en cuenta. 
Finalmente, debe ser tomado en cuenta que existe un gran número de economías regionales contiguas. Cada una es diferente de la otra en términos de estructura y base económica. La fuente de gastos autónomos y la magnitud de los parámetros diferirá. De este modo, si los parámetros de este modelo fuesen estimados para una región, estos no serían necesariamente aplicables a otras regiones. Para que tengan sentido para toda la frontera, el modelo deberá ser aplicado independientemente en cada región, lo que implica un proceso muy costoso.

\section{CONCLUSION}

El modelo aquí desarrollado y varios otros, como los modelos de ingreso-egreso, pueden ser útiles en la descripción de las economías de las regiones fronterizas contiguas. Ellos son importantes como marcos conceptuales en la comprensión de la complejidad e interrelatividad de dos economías. Sin embargo, la aplicación de dichos modelos para predecir cambios en la actividad económica, resultantes de la implementación de nuevas políticas o condiciones económicas, requieren una cantidad considerable de datos muy costosos tanto en tiempo como en dinero, además de que, para ser válidos, deben ser puestos al día regularmente.

La falta de datos adecuados y el alto costo de su obtención son las razones para la brecha existente entre la teoría de las economías fronterizas y las aplicaciones de esa teoría para mostrar el impacto cuantitativo de políticas económicas nuevas o anteriormente aplicadas y las condiciones en ambas regiones contiguas. Como las economías contiguas se están agrandando y volviéndose cada vez más importantes para las dos naciones, sería muy útil ser capaz de predecir el impacto de las políticas económicas y las condiciones en las regiones. Sin embargo, hacer eso requeriría gastos significativos en la obtención de datos. Se espera que el reciente establecimiento de bancos de datos fronterizos en la Universidad Estatal de San Diego, el Centro de-Estudios Fronterizos del Norte de México (CEFNOMEX) y otros lugares, facilite la recolección de información continua y regularmente, de modo que sea eliminada la brecha entre la teoría y su aplicación. 